Año 13.

Académica sin Frontera

Núm. 34 Tema COVID 19

Especial

ISSN: 2007-8870

https://revistainvestigacionacademicasinfrontera.unison.mx/index.php/RDIASF

Recibido el 15 de agosto de 2020. Dictaminado mediante arbitraje favorablemente 11 de diciembre de 2020.

\title{
EI emprendimiento frente a la pandemia provocada por el COVID-19
}

\author{
Dra. Patricia Aguilar Talamante \\ patricia.aguilar@unison.mx \\ ORCID: 0000-0002-7270-0220 \\ Dr. José Alfredo Heredia Bustamante \\ alfredo.heredia@unison.mx \\ ORCID: 0000-0001-5691-1645 \\ M.A. Nora Gabriela Sainz Zarate \\ nora.sainz@unison.mx \\ ORCID: 0000-0001-5685-1654
}

\begin{abstract}
Resumen
Proteger el emprendedurismo preservando su capital humano y estructura organizacional, así como su capacidad innovadora, permitirá acelerar la reconstrucción económica y social para la salida de la crisis de cualquier pais. Esta investigación, por su diseño es descriptiva y exploratoria y su objetivo es determinar, a partir de la apreciación que tienen los emprendedores del sector comercio minoritario de Hermosillo, Sonora cuál es el impacto, reacciones y redes de apoyo del emprendimiento frente a la crisis del COVID19. Los resultados obtenidos en la investigación muestran que 8 de cada 10 emprendimientos manifiestan estar siendo fuertemente afectados por la crisis. En conclusión, el emprendimiento después de la pandemia, necesita como plan de acción una planificación estratégica administrativa para la toma de decisiones que ayude a generar modelos de negocios capaces de enfrentar una crisis.
\end{abstract}

Palabras claves: Impacto, COVID-19, Crisis, Planeación Estratégica, Redes de Apoyo. 


\title{
Entrepreneurship front the face of the pandemic caused by COVID-19.
}

\begin{abstract}
Protecting entrepreneurship while preserving its human capital and organizational structure, as well as its innovative capacity, will allow to accelerate the economic and social reconstruction for the exit from the crisis of any country. This research, due to its design, is descriptive and exploratory and its objective is to determine, based on the appreciation that entrepreneurs of the minority trade sector of Hermosillo, Sonora have what is the impact, reactions and support networks of entrepreneurship in the face of the crisis of the COVID-19. The results obtained in the research show that 8 out of 10 enterprises indicate that they are being strongly affected by the crisis. In conclusion, entrepreneurship after the pandemic needs as an action plan administrative strategic planning for decision-making that helps generate business models capable of facing a crisis.
\end{abstract}

Key words: Impact, COVID-19, Crisis, Strategic Planning, Support Networks.

\section{Introducción}

En el 2019 las pequeñas y medianas empresas (pymes) en México se preparaban para un año 2020 con una serie de medidas y cambios legislativos que podrían afectar su crecimiento y, muy probablemente su permanencia en el mercado, como lo menciona Pérez (2019) en revista Forbes 2019 y, señala tres de los puntos que estaban incluídos en el paquete económico 2020:

1. En el ámbito fiscal, se afecta su competitividad al pretender gravar a las actividades de plataformas digitales con una retención de entre el 2 y $17 \%$ en IVA, y retenciones del IRS del 2 al 8\%, incluye personas, transporte y entregas.

2. Disminución del $32 \%$ en el presupuesto de egresos, ya que únicamente se contempla 1,929 mdp comparado con los 4,314 que se asignaron en el 2019. Se dará impulso a programas financieros con tasas preferenciales buscando apoyar a 130,000 pymes y 370,000 micronegocios (mipymes) con financiamientos de hasta 270 mil mdp.

3. Sin embargo, la controversia se centra en la tipificación como delito grave de la emisión de facturas falsas, medida con lo que el gobierno federal espera recuperar hasta el $30 \%$ de los 80 mil millones de dólares que se pierden en las Finanzas Públicas. 
Año 13.

Núm. 34 Tema COVID 19

Especial
Académica sin Frontera

ISSN: 2007-8870

\section{$\underline{\text { https://revistainvestigacionacademicasinfrontera.unison.mx/index.php/RDIASF }}$}

Recibido el 15 de agosto de 2020. Dictaminado mediante arbitraje favorablemente 11 de diciembre de 2020.

En junio 2020 el Banco Mundial (World Bank, 2020) liberó un sombrío reporte en el cual prevé una contracción del Producto Interno Bruto (PIB) mundial para este año del 5.2 por ciento, el mayor en ocho décadas, alertando del empeoramiento de los pronósticos en la medida que se prolongue el tiempo para controlar la pandemia o se produzcan efectos financieros en cascada. Lo anterior, muestra un panorama de incertidumbre y complejidad al que tienen que enfrentarse más de 4 millones de pymes en México, ante la pandemia del COVID-19, lo cual hace difícil para grandes compañías porque puede resultar mortal para estas empresas que son el motor de México, al generar el 72\% del empleo y alrededor del 52\% de nuestro PIB.

La crisis económica causada por el COVID-19 hizo que los gobiernos de los países de América Latina y el Caribe desarrollen nuevas políticas públicas y planes de reactivación económica adoptando enfoques diferentes para manejar los mercados laborales, aumentando el uso de internet, emprendimientos que se han adaptado a la pandemia, enfrentando nuevos desafíos para navegar con éxito en su crecimiento, como la comunicación, entretenimiento y compras en línea (Bonaglia, Nieto, y Vásquez, 2020; Donthu y Gustafsson, 2020).

Esta crisis trajo consigo grandes retos a los que tuvieron que hacer frente las diferentes economías: las nuevas reformas laborales, la necesidad de aumento de productividad empresarial, la recuperación de puestos de trabajo, encontrar nuevos nichos y vías de negocios, las tecnologías de la información y la comunicación, el proceso de globalización, la creciente competencia en los mercados, la aparición de mercados emergentes, pero dentro de todo este caos, algo que es impotante reconocer es que el emprendimiento parte de un capital humano creativo e innovador y aunado a la diversidad tecnológica, se deben aprovechar las formas en que los individuos satisfacen las necesidades para generar nuevos emprendimientos, las cuales son demasiado numerosas para enumerarlas por completo, pero está claro que a través de la digitalización ha 
sido posible satisfacer las mismas en el periodo de confinamiento vivido (Haeffele, Hobson y Storr, 2020).

El emprendimiento se vincula con la innovación, porque aporta al desarrollo económico, está intimamente relacionado con la flexibilidad y el conocimiento, factores que son fuente de competitividad en una economía mundial globalizada, donde la transformación tecnológica y la competencia global cada vez es más intensa, lo que provoca la necesidad de el gobierno sustente politicas encaminadas a fomentar el emprendimiento y resolver sus problemáticas a partir de la crisis actual (Sornoza, Parrales, Cañarte, Castillo, Guaranda, Delgado, 2018; Sumba, Almendaris, Baque y Aliatis, 2020).

El papel del emprendedor ha sido, es y será un recurso fundamental en periodos de crisis, en los que se dan oportunidades para renovar la economía de un territorio a través de su papel. De ahí que, ante esta crisis provocada por la COVID-19, lo que se tiene que hacer sea promover la investigación en emprendimiento con el fin de tener la información necesaria con la que se desarrollen políticas eficientes en nuestro país que promuevan este necesario fenómeno empresarial, el cual provocará la regeneración económica del país y, por tanto, la salida de la crisis (Martínez y Rubio, 2020). Partiendo de lo anterior, el objetivo del presente estudio pretende determinar, bajo la perspectiva de los emprendedores del sector comercio minorista de Hermosillo, Sonora cuál es el impacto, reacciones y redes de apoyo del emprendimiento ante la crisis del COVID-19 que permita proponer un plan de acción a futuro.

\section{Materiales y Método}

En este documento se presentan los avances de una investigación de campo bajo un enfoque metodológico descriptivo y de corte transversal con el objetivo de determinar, bajo la perspectiva de los emprendedores del sector comercio minorista de Hermosillo, Sonora cuál es el impacto, reacciones y redes de apoyo del emprendimiento ante la crisis del COVID-19. Para este estudio se utilizó un cuestionario estandarizado (validado a juicio de expertos y con un coeficiente de confiabilidad Alpha de Cronbach de 0,92 Muy Alta) por (González-Díaz \& Perez, 2015) a 150 emprendedores del sector comercio minorista de la ciudad de Hermosillo, Sonora, con una fuerza laboral entre 10 y 250 trabajadores. El instrumento contiene 15 items dividido en tres variables; Variable 1; Impactos al emprendimiento, Variable 2; Reacciones de los emprendedores y, 
Año 13.

Núm. 34 Tema COVID 19

Especial
Revista de Investigación

Académica sin Frontera

ISSN: 2007-8870

\section{https://revistainvestigacionacademicasinfrontera.unison.mx/index.php/RDIASF}

Recibido el 15 de agosto de 2020. Dictaminado mediante arbitraje favorablemente 11 de diciembre de 2020.

Variable 3; Redes de apoyo en los emprendimientos durante la crisis del COVID-19, y su aplicación se llevó a cabo mediante un Google form (González-Díaz et al., 2016).

\section{Resultados}

Tras analizar los datos obtenidos por medio de los estudios exploratorios, a continuación, se presentan los resultados más relevantes del trabajo de investigación basado en la fase exploratoria de la investigación, donde se muestra la opinión de los emprendedores del sector comercio minorista de la ciudad de Hermosillo, Sonora elegidas, en referencia a el impacto, reacciones y redes de apoyo del emprendimiento ante la crisis del COVID-19.

Imagen 1. Tiempo en el mercado

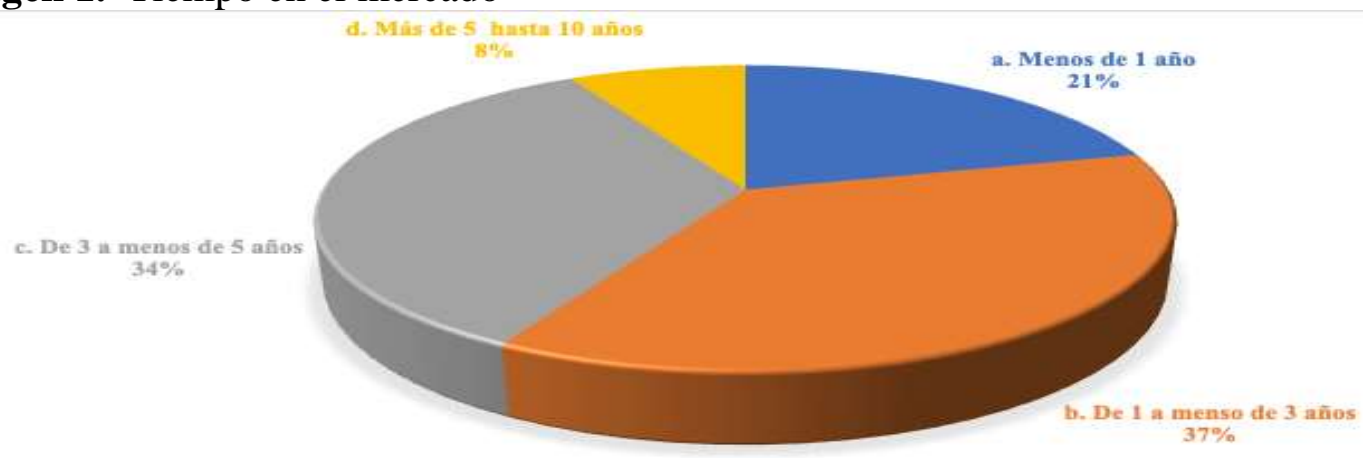

Fuente: Elaboración propia en base a resultados de la investigación.

La imagen 1, muestra que el $37 \%$ de la muestra tiene de 1 a menos de 3 años en el mercado, el $21 \%$ tiene menos de 1 año, el $34 \%$ tiene de 3 a menos de 5 años y un $8 \%$ más de 5 hasta 10 años. 


\section{Variable 1; Impactos al emprendimiento}

Imagen 2. Tipos de Impacto

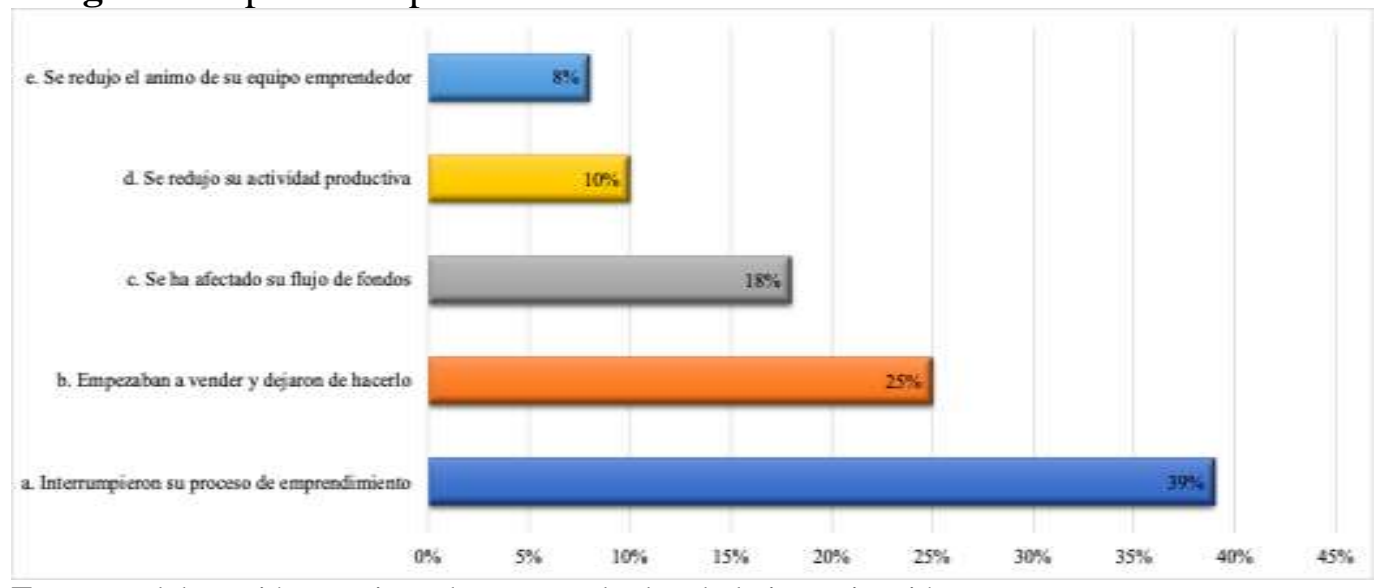

Fuente: Elaboración propia en base a resultados de la investigación.

En la Imagen 2, se muestran los tipos de impactos del COVID-19 al emprendimiento. El $39 \%$ de los emprendedores encuestados, que aún no habían puesto en marcha su emprendimiento interrumpieron el proceso, el 25\% empezaban a vender y dejaron de hacerlo, el 18\% menciona que ha afectado su flujo de fondos, el $10 \%$ menciona que se redujo su actividad productiva y el $8 \%$ que se redujo el animo de su equipo emprendedor.

\section{Variable 2; Reacciones de los emprendedores}

Imagen 3. Como prevén financiarse ante la crisis

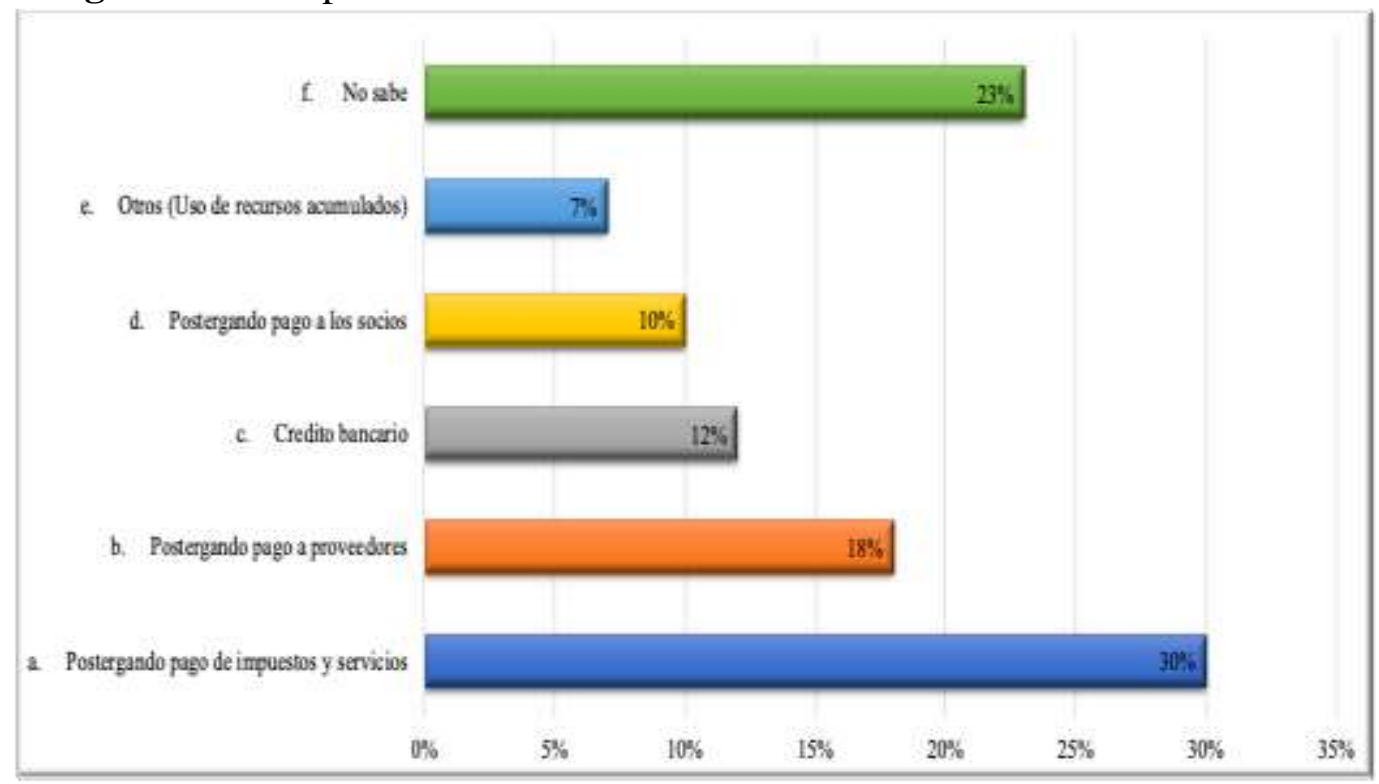

Fuente: Elaboración propia en base a resultados de la investigación. 

"Fl suber ile mis hijos
hardi mi pronderis"

Año 13.

Núm. 34 Tema COVID 19

Especial

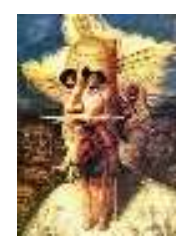

Revista de Investigación

Académica sin Frontera

ISSN: 2007-8870 https://revistainvestigacionacademicasinfrontera.unison.mx/index.php/RDIASF

Recibido el 15 de agosto de 2020. Dictaminado mediante arbitraje favorablemente 11 de diciembre de 2020.

En la Imagen 3, se muestra que el $30 \%$ prevé financiarse ante la crisis postergando el pago de impuestos y servicios, el $23 \%$ no sabe, el $18 \%$ postergando pago a proveedores, el $12 \%$ mediante crédito bancario, el $10 \%$ postergando pago a socios y el $7 \%$ mediante otros tales como el uso de recursos acumulados.

Imagen 4. Nivel de organización del trabajo

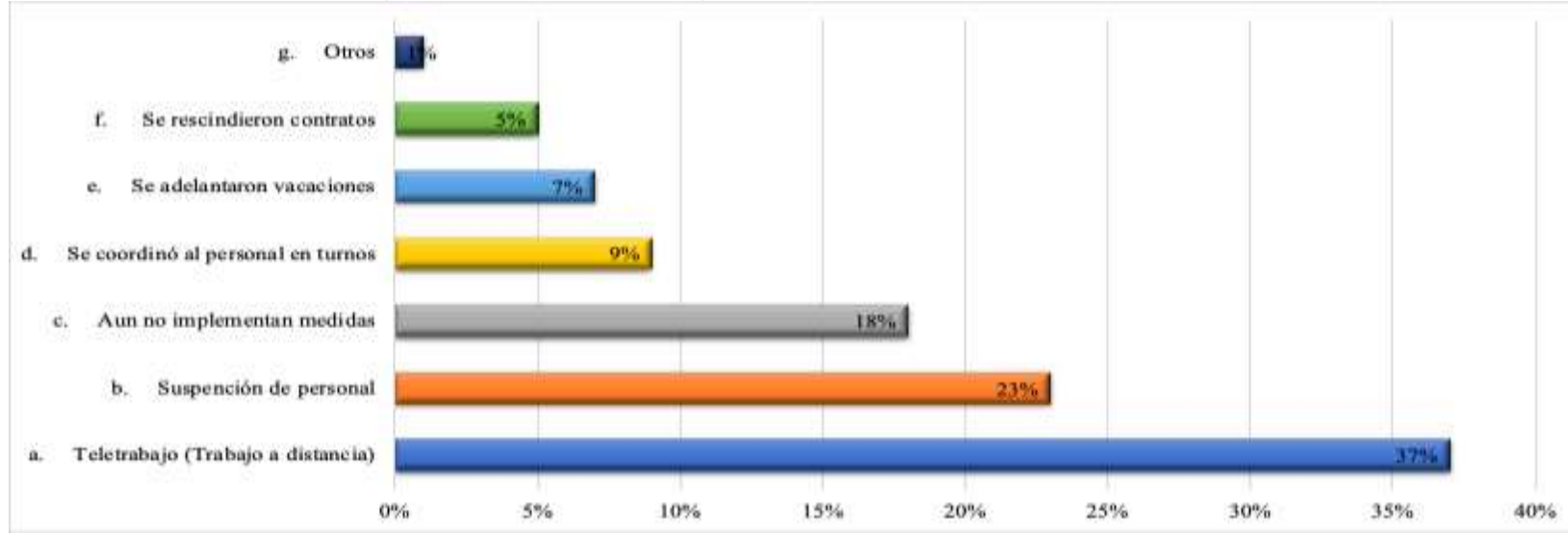

Fuente: Elaboración propia en base a resultados de la investigación.

En la Imagen 4, se observa el nivel de organización del trabajo. Para el 37\% el nivel de trabajo es mediante Teletrabajo (Trabajo a distancia), el 23\% mediante suspensión de personal, el $18 \%$ aun no implementa medidas, el $9 \%$ se coordinó con el personal en turnos, el 7\% adelantó trabajo, el $5 \%$ se rescindieron contratos y el $1 \%$ otros tales como despidos forzosos. 


\section{Variable 3; Redes de apoyo en los emprendimientos durante la crisis del COVID-19}

Imagen 5. A quien acuden para responder ante la crisis

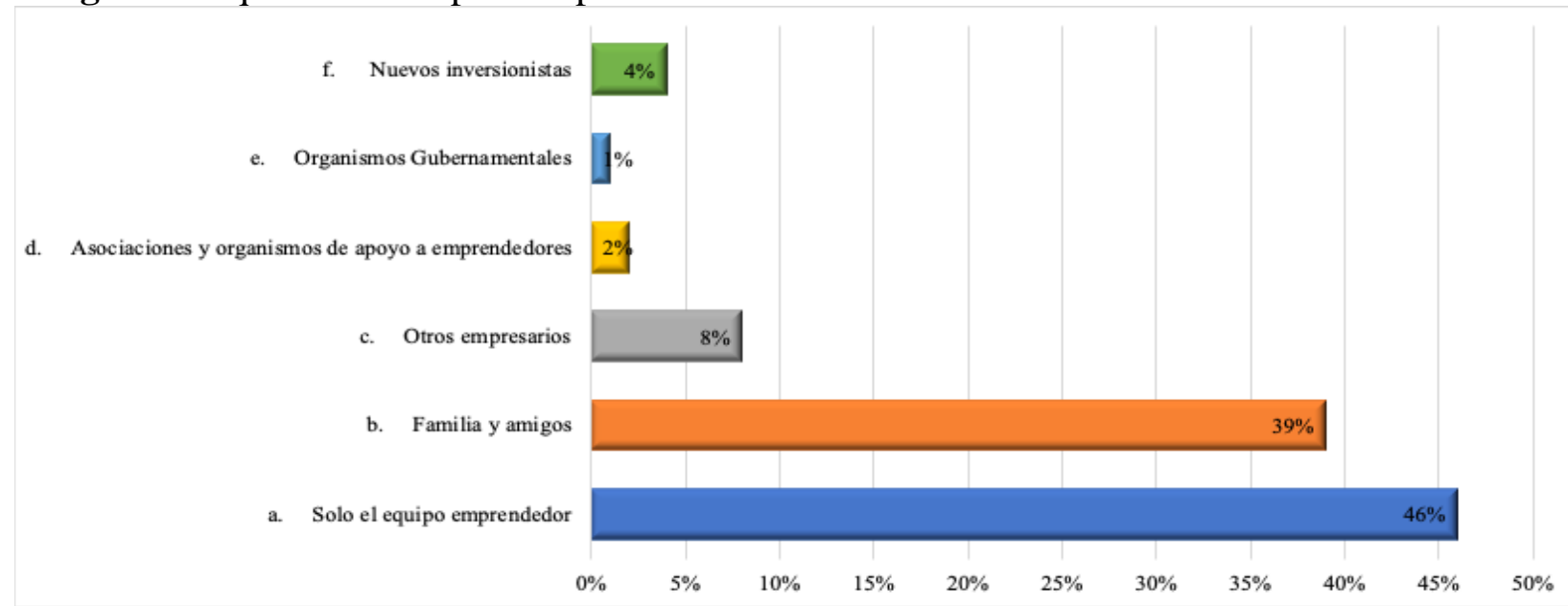

Fuente: Elaboración propia en base a resultados de la investigación.

En la imagen 5, se muestra la información relacionada con las redes de apoyo en los emprendimientos durante el COVID-19. El 46\% utiliza solo el equipo emprendedor para responder a la crisis, el 39\% acude a la familia y amigos, el $8 \%$ a otros empresarios, el $4 \%$ a nuevos inversionistas, el $2 \%$ a asociaciones y organismos de apoyo a empresarios y el $1 \%$ a Organismos Gubernamentales.

\section{Discusión de resultados}

Los resultados de la investigación muestran que el impacto en los emprendimientos y las empresas es notable, en especial en las empresas en proceso de gestación y las jóvenes consideradas como aquellas de menos de 3 años en el mercado. Más de la mitad de los emprendedores encuestados que aun no habían puesto en marcha su emprendimiento interrumpieron su proceso y los que ya estaban establecidos sufrieron caídas de ventas considerables. De las reacciones de los emprendedores durante la crisis provocada por el COVID-19, se observa que se prevé financieramente mediante estrategias comunes de retraso de pago de impuestos y proveedores. Pero también existe un grupo que aún no tiene claro como financiará la crisis. En referencia a la reacción relacionada con la organización del trabajo, optan por el trabajo a distancia, pero se sienten preocupados porque no están preparados para ello y, otra de sus estrategias de organización es suspender a su personal durante la crisis para 
Año 13.

Núm. 34 Tema COVID 19

Especial
Académica sin Frontera

ISSN: 2007-8870

\section{https://revistainvestigacionacademicasinfrontera.unison.mx/index.php/RDIASF}

Recibido el 15 de agosto de 2020. Dictaminado mediante arbitraje favorablemente 11 de diciembre de 2020.

protegerlos. En cuanto a las redes de apoyo, la mayoría de los emprendedores solo cuenta con el apoyo de su equipo emprendedor y, en menor medida, de su red social.

\section{Conclusiones}

La pandemia provocada por el COVID-19 está generando impactos a nivel humanitario como también en las actividades económicas. Es notable que entre aquellos grupos afectados se encuentran los emprendimientos y en especial las empresas en proceso de gestión o las jóvenes por lo que es crucial la atención que se pueda brindar mediante la implementación de estrategias que contemplen su situación.

La crisis generada por la pandemia ha traído consigo un panorama de enorme incertidumbre económica y jurídica, cuyos efectos solo son mitigables a través de medidas capaces de dar respuesta a las múltiples necesidades urgentes que tienen las empresas. Los resultados que muestran la presente investigación, hacen ver desde la perspectiva del emprendedor del sector comercio minorista de la ciudad de Hermosillo, Sonora cual es el impacto, reacciones y redes de apoyo del emprendimiento ante la crisis del COVID-19, información que pone de manifiesto, la necesidad de generar un plan de acción a futuro que contenga estrategias que contemple los resultados aquí encontrados.

A medida que pasa el tiempo, las empresas buscan ajustarse a la nueva normalidad, pero las respuestas desde las organizaciones de apoyo al emprendedurismo tardan en llegar y, aun así, no terminan de ajustarse a sus demandas, porque muchas de estas organizaciones han detenido o disminuido significativamente el trabajo con los emprendedores. Por su parte estos, dentro de sus principales problemas incluyen la falta de servicios apropiados para atender a sus necesidades en la emergencia, junto con la caída de los ingresos y la incertidumbre ante el futuro, lo cual sugiere bajos niveles de resiliencia y flexibilidad organizacional en los ecosistemas emprendedores. 
La cultura emprendedora es clave como agente de cambio, como visión de cómo vamos transformándonos como sociedad en cuanto a nuestras necesidades y a los cambios tecnológicos que nos hacen también transformar la manera en que consumimos, por eso pensamos que esta cultura crea también una distinción porque no necesariamente significa que la gente se haga empresaria, no importa si van a un empleo o generan su propia empresa, pero van con la mentalidad de ser agentes de cambio.

Si bien es cierto, la pandemia provocada por el COVID-19 ha hecho que la empresa enfrente un enorme reto desde diferentes perspectivas, es importante hacer un alto y recordar que los cambios son una constante, y esto no va a cambiar, pero lo que si se puede hacer es aprender de las experiencias de otras épocas y de otras empresas. Los resultados de la presente investigación, ponen de manifiesto que el emprendimiento después de la pandemia, necesita como plan de acción la planificación estratégica administrativa para mejorar la toma de decisiones e incrementar la rentabilidad, mediante estrategias que generen modelos de negocio basado en un sustento preparado para enfrentar los desafíos que generan un cambio.

\section{Referencias}

Alaña Castillo, T. P., Crespo García, M. K., \& Gonzaga Añazco, S. J. (2018). ¿Cómo las estrategias empresariales permiten una ventaja competitiva en las micro, pequeñas y medianas empresas de la provincia de El Oro? Revista Universidad y Sociedad, 10(2), 257-262.

Bonaglia, F., Nieto, S., y Vásquez, J. (2020). Una mirada al futuro post-COVID-19: hacia un nuevo pacto social en América Latina y el Caribe. Análisis Carolina (21), 1-15. doi:https://doi.org/10.33960/AC_21.2020.

Donthu, N., y Gustafsson, A. (2020). Effects of COVID-19 on business and research. Journal of Business Research, 117, 284-289. doi:https://doi.org/10.1016/j.jbusres.2020.06.008

Haeffele, S., Hobson, A., y Storr, V. H. (2020). Coming Back from Covid-19: Lessons in Entrepreneurship from Disaster Recovery Research.SSRN Electronic Journal. https://doi.org/10.2139/ssrn.3592966

Nicolás Martínez, C., y Rubio Bañón, A. (2020). Entrepreneurship in times of crisis: An exploratory analysis of the COVID-19's effects. Small Business International Review, 4(2), 53-66. https://doi.org/10.26784/sbir.v4i2.279 
"Fl sibler ite mis hijise

Año 13.

Núm. 34 Tema COVID 19

Especial

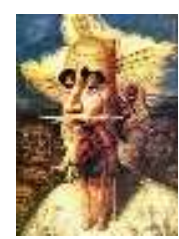

Revista de Investigación

Académica sin Frontera

ISSN: 2007-8870

https://revistainvestigacionacademicasinfrontera.unison.mx/index.php/RDIASF

Recibido el 15 de agosto de 2020. Dictaminado mediante arbitraje favorablemente 11 de diciembre de 2020.

Pérez, E. (2019). "Pymes, con panorama complicado para 2020" en Forbes, México. 23 octubre de 2020. Accesado el 8 de noviembre de 2020, de https://www.forbes.com.mx/pymesconpanorama-complicado-para-2020/

Sornoza Parrales, D. R., Parrales Poveda, M. L., Sornoza, G., Cañarte, T., \& Castillo, A. (2018). Fundamentos de Emprendimientos. Área de Innovación y Desarrollo, S.L. doi: http://dx.doi.org/10.17993/EcoOrgyCso.2018.39

Sumba Bustamante, R. Y., Almendariz Gonzalez, S. M., Baque Chancay, C. L. y Aliatis Bravo, V. G. (2020). Emprendimientos en tiempo de covid-19: De lo tradicional al comercio electrónico. Revista Científica FIPCAEC (Fomento De La investigación Y publicación En Ciencias Administrativas, Económicas Y Contables). ISSN : 2588-090X . Polo De Capacitación, Investigación y Publicación (POCAIP), 5(4), 137-164. https://doi.org/10.23857/fipcaec.v5i4.300

World Bank. 2020. Global Economic Prospects, June 2020. Washington, DC. Recuperado de https://www.bancomundial.org/es/news/press-release/2020/06/08/covid-19-toplungeglobal-economy-into-worst-recession-since-world-war-ii 


\section{Directorio Institucional}

Dr. Enrique Fernando Velázquez Contreras

Rector

Dr. Ramón Enrique Robles Zepeda

Secretario General Académico

Dra. Rosa María Montesinos Cisneros

Secretaria General Administrativa

Dr. Rodolfo Basurto Álvarez

Director de Vinculación y Difusión

Dra. Adriana Leticia Navarro Verdugo

Vicerrectora de la Unidad Regional Sur

Dr. Ernesto Clark Valenzuela

Director de la División de Ciencias Económicas y Sociales

Dr. Francisco Espinoza Morales

Secretario de la División de Ciencias Económico y Sociales

Dra. Leticia María González Velásquez

Jefe del Departamento de Ciencias Económico Administrativas

Dra. Lidia Amalia Zallas Esquer

Jefe de Departamento de Ciencias Sociales 
Año 13.

Núm. 34 Tema COVID 19

Especial

ISSN: 2007-8870

https://revistainvestigacionacademicasinfrontera.unison.mx/index.php/RDIASF

Recibido el 15 de agosto de 2020. Dictaminado mediante arbitraje favorablemente 11 de diciembre de 2020.

\section{Comité Directivo}

\section{Editor Responsable}

Dr. Francisco Espinoza Morales

Universidad de Sonora

Directora

Dra. Leticia María González Velásquez

Universidad de Sonora

Subdirector

Dr. Javier Carreón Guillen

Universidad Nacional Autónoma de México

Editor Científico

Dr. Cruz García Lirios

Universidad Autónoma del estado de México

Master Gráfico

M.T.I. Francisco Alan Espinoza Zallas

Universidad Estatal de Sonora

Nos complace anunciar que su diario, "Academic Research Journal Withoutborders" (ISSN/EISSN 20078870) fue evaluado positivamente en la indexación Citefactor, ahora la página de la revista está disponible en línea, en caso de cualquier problema.

Journals Master | International Innovative Journal Impact Factor (IIJIF)

Red Latinoamericana de revistas Académicas en Ciencias Sociales y Humanidades
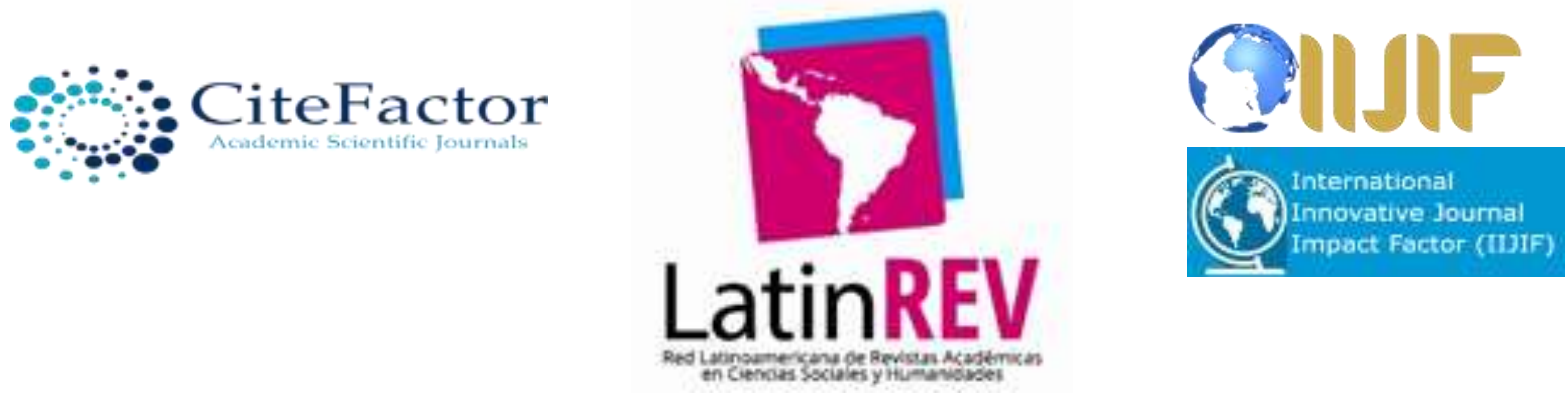


\section{Comité editorial}

Dra. Angélica María Rascón Larios

Universidad de Sonora. México

Dra. María del Rosario Molina González

Universidad de Sonora

Dra. Francisca Elena Rochin Wong

Universidad de Sonora. México

Dra. Lidia Amalia Zallas Esquer

Universidad de Sonora. México

Dra. Beatriz Llamas Arechiga

Universidad de Sonora. México

Dr. Rogelio Barba Álvarez

Universidad de Guadalajara. México

Dra. Rosa María Rincón Ornelas

Universidad de Sonora. México

Dr. Juan Flores Preciado

Universidad de Colima. México

Dr. Amado Olivares Leal. Universidad de Sonora

Universidad de Sonora. México

Dr. Guillermo Velázquez Valadez.

Instituto Politécnico Nacional (IPN) México

Dr. Hugo Nefstalí Padilla Torres.

Universidad Estatal de Sonora. México

Dr. Luis Ramón Moreno Moreno.

Universidad Autónoma de Baja California. México

Dr. Miguel Ángel Vázquez Ruiz.

Universidad de Sonora. México

Dra. Lorena Vélez García.

Universidad Autónoma de Baja California. México

Dra. Pabla Peralta Miranda.

Universidad Simón Bolívar, Barranquilla, Colombia

Mtro. Roberto Espíritu Olmos

Universidad de Colima (FCA Tecomán) Colima 
Año 13.

Académica sin Frontera

Núm. 34 Tema COVID 19

Especial

ISSN: 2007-8870

https://revistainvestigacionacademicasinfrontera.unison.mx/index.php/RDIASF

Recibido el 15 de agosto de 2020. Dictaminado mediante arbitraje favorablemente 11 de diciembre de 2020.

\author{
Dr. Héctor Priego Huertas. \\ Universidad de Colima (FCA Tecomán) Colima \\ Mtra. María Guadalupe Alvarado Ibarra. \\ Universidad de Sonora. México. \\ MSc. Celso Germán Sánchez Zayas \\ Universidad de Camagüey, Ignacio Agramonte Loynaz, Cuba \\ Dra. María Luisa Quintero Soto \\ Universidad Autónoma del Estado de México \\ Dr. Eyder Bolivar Mojica \\ Universidad Católica, Luis Amigó, Medellin, Colombia

\section{Revisores de Textos en Inglés} \\ Mtro. Renato Encinas \\ Mtra. Cecilia Guadalupe Martínez Solano
}

\title{
Comité científico
}

Dr. Rosendo Martínez Jiménez. Universidad Autónoma Benito Juárez de Oaxaca.

Dr. Hugo Neftalí Padilla. Universidad Estatal de Sonora

Dra. María Teresa Gaxiola Sánchez. Universidad de Sonora.

Dr. José Cesar Kaplan. Universidad Estatal de Sonora.

Dr. Alfredo Islas Rodríguez. Universidad de Sonora

Frecuencia de publicación: semestral / 2 números por año. 
Revista de Investigación Académica sin Frontera (RIASF) con (ISSN: 2007-8870) es un interlocutor internacional de acceso abierto revisado diario en línea en el ámbito del de las Ciencias Económicas Administrativas y Sociales. Su objetivo principal es dar a los trabajos de investigación de calidad. Cubre todas las sub-campos de los campos anteriormente mencionados. Proporciona la plataforma a académicos, estudiantes y profesionales. Sólo pública trabajos de investigación y artículos de revisión inicial. Documento presentado debe cumplir con algunos criterios como, debe ser original, inédita y no estén sometidos a ninguna otra revista.

RIASF es una revista arbitrada / Revisión por pares International. Publicamos documentos sobre una variedad de temas, contextos y estrategias de análisis que examinan la relación entre la rápida evolución para la Sociedad y la tecnología del conocimiento.

REVISTA DE INVESTIGACIÓN ACADÉMICA SIN FRONTERA, Año 13, No. 34, especial, Julio diciembre 2020, es una publicación semestral de investigación científica, editada por la Universidad de Sonora, a través de las División de Ciencias Económicas y Sociales, de la Unidad Regional Sur, Blvd. Lázaro Cárdenas No. 100, Col. Francisco Villa, Navojoa, Sonora, Sonora, México, C.P. 85880. Tel. (642) 425- 99-54. https://revistainvestigacionacademicasinfrontera.unison.mx/index.php/RDIASF

revistaacademicasinfrontera@unison.mx.

Editor responsable: Francisco Espinoza Morales. Reserva de Derechos al Uso Exclusivo: 04-2013121811323700-203 e ISSN: 2007-8870, ambos otorgados por el Instituto Nacional de Derecho de Autor. Inscrita en el Directorio de LATINDEX, con Núm. De folio 20014, folio único 14590. Responsable de la última actualización de este Número, Unidad Informática de la Universidad de Sonora, fecha de la última modificación, 30 de diciembre 2020, indexada a Cite Factor Academic Scientific Journal y Journals Master (IIJIF) y Red Latinoamericana de Revistas Académicas en Ciencias Sociales y Humanidades, (Latín Rev). Las opiniones expresadas por los autores no necesariamente reflejan la postura del editor de la publicación. Se autoriza la reproducción total o parcial de los contenidos e imágenes en la presente publicación siempre y cuando se cuente con la autorización del editor y se cite plenamente la fuente. 


\section{Año 13.}

Núm. 34 Tema COVID 19

Especial
Revista de Investigación

Académica sin Frontera

ISSN: 2007-8870

https://revistainvestigacionacademicasinfrontera.unison.mx/index.php/RDIASF

Recibido el 15 de agosto de 2020. Dictaminado mediante arbitraje favorablemente 11 de diciembre de 2020.

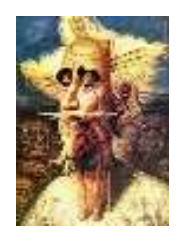

Nos complace anunciar que su diario, "Academic Research Journal Withoutborders" (ISSN/EISSN 20078870) fue evaluado positivamente en la indexación Citefactor, ahora la página de la revista está disponible en línea, en caso de cualquier problema.

Journals Master | International Innovative Journal Impact Factor (IIJIF)

\section{Red Latinoamericana de revistas Académicas en Ciencias Sociales y Humanidades}

\section{CiteFactor}

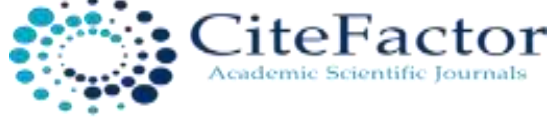

\section{ESJI Injim www.ESJIndex.org}
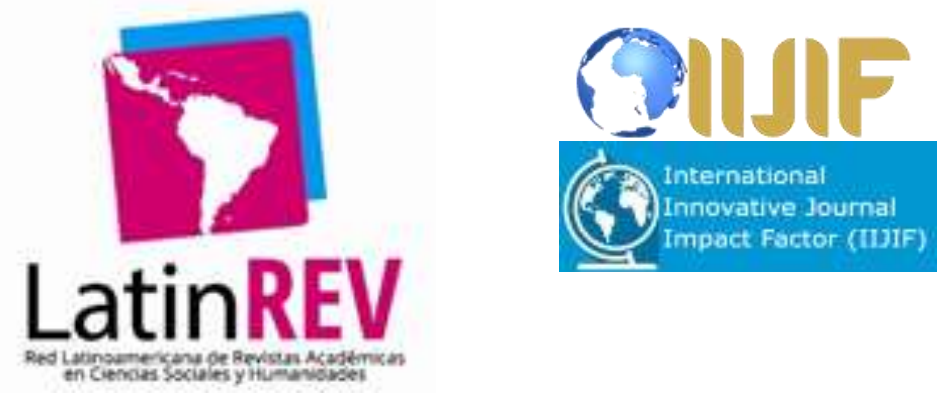

https://www.neliti.com
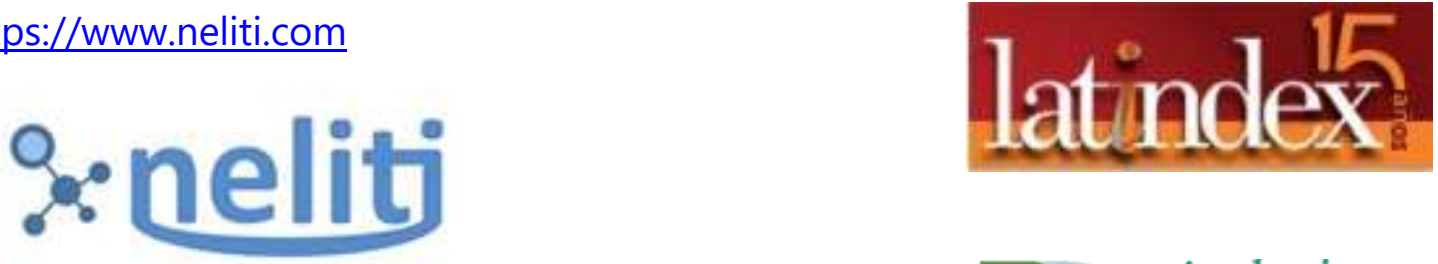

Indonesia's Research Repository

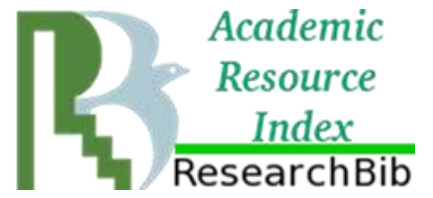


